\title{
A Matrix Formulation for the Moment Distribution Method Applied to Continuous Beams
}

\author{
Arlindo Pires Lopes, ${ }^{1,2}$ Adriana Alencar Santos, ${ }^{2}$ and Rogério Coelho Lopes ${ }^{2}$ \\ ${ }^{1}$ Department of Civil Engineering, Portland State University, Portland, OR 97201-3252, USA \\ ${ }^{2}$ Coordenação de Engenharia Mecânica, Escola Superior de Tecnologia, Universidade do Estado do Amazonas, \\ Avenida Darcy Vargas 1200, 69065-020, Manaus, AM, Brazil
}

Correspondence should be addressed to Arlindo Pires Lopes, lopesa@cecs.pdx.edu

Received 8 January 2011; Accepted 14 February 2011

Academic Editor: Farid Taheri

Copyright (c) 2011 Arlindo Pires Lopes et al. This is an open access article distributed under the Creative Commons Attribution License, which permits unrestricted use, distribution, and reproduction in any medium, provided the original work is properly cited.

The Moment Distribution Method is a quite powerful hand method of structural analysis, in which the solution is obtained iteratively without even formulating the equations for the unknowns. It was formulated by Professor Cross in an era where computer facilities were not available to solve frame problems that normally require the solution of simultaneous algebraic equations. Its relevance today, in the era of personal computers, is in its insight on how a structure reacts to applied loads by rotating its nodes and thus distributing the loads in the form of member-end moments. Such an insight is the foundation of the modern displacement method. This work has a main objective to present an exact solution for the Moment Distribution Method through a matrix formulation using only one equation. The initial moments at the ends of the members and the distribution and carry-over factors are calculated from the elementary procedures of structural analysis. Four continuous beams are investigated to illustrate the applicability and accuracy of the proposed formulation. The use of a matrix formulation yields excellent results when compared with those in the literature or with a commercial structural program.

\section{Introduction}

It is observed in practice that the greater the complexity of a structure, the greater the number of unknowns and therefore the greater the number of simultaneous equations requiring solution. Hand methods of analysis then become extremely tedious if not impracticable, so that alternatives are desirable. One obvious alternative is to employ computerbased techniques, but another quite powerful hand method is an iterative procedure known as the moment distribution method (MDM).

The MDM for the analysis of rigid-jointed structures was introduced by Professor Hardy Cross over seventy five years ago. In the past, when structural engineers had to compute the forces and displacements in a statically indeterminate structure, they inevitably turned to what was generally known as the Hardy Cross method. In this method, the fixed-end moments in the framing members are gradually distributed to adjacent members in a number of steps, such that the system eventually reaches its natural equilibrium configuration.

The method is still taught in every undergraduate civil engineering course on the analysis and design of engineering structures, and although the Hardy Cross Method has been superseded by more powerful procedures such as the finite element method, the MDM made possible the efficient and safe design of many reinforced concrete buildings during an entire generation.

The original paper written by Cross [1] explaining the method was followed afterwards by a famous textbook written by Cross and Morgan [2]. In the former one, he wrote the following: "The essential idea which the writer wishes to present involves no mathematical relations except the simplest arithmetic." The MDM depends on the solution of three problems for beam constants: the determination of fixed-end moments, of the stiffness at each end of a beam, and of the 

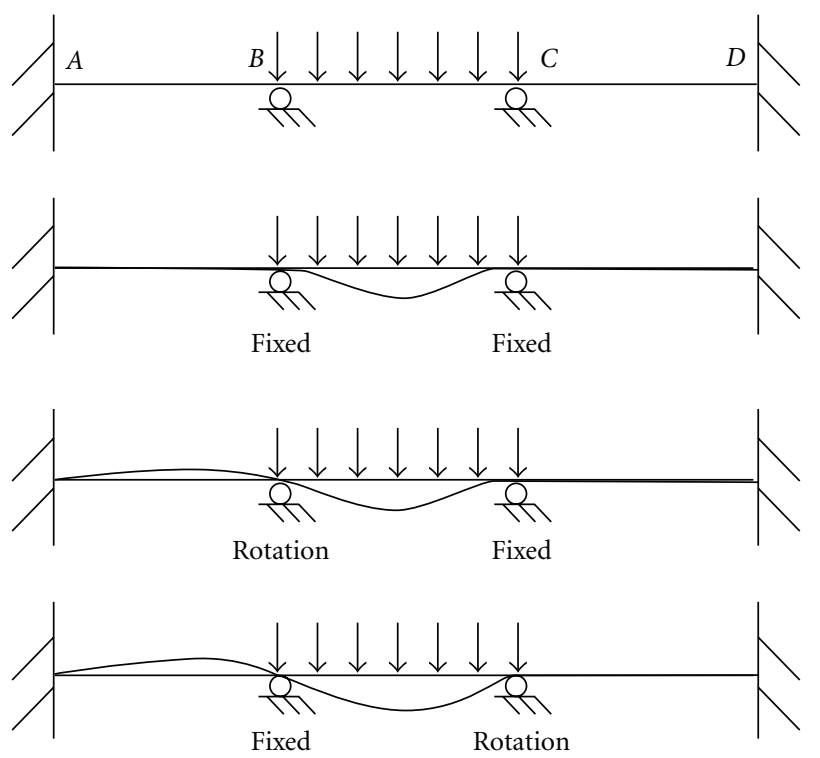

FIgUre 1: Continuous beam subjected to static loads.

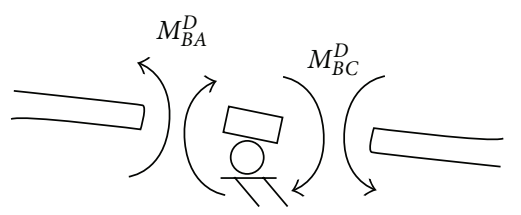

FIGURE 2: Free-body diagram of node $B$.

carry-over factor at each end for every member of the structure under consideration, which will be explained afterwards.

Lightfoot [3] believes that the simpler possibilities of the MDM have now all been discovered and that its economical use for the analysis and design of complex structures must now be assessed in relation to available electronic computing programs and facilities.

The purpose of this paper is to enhance and improve the MDM used in solving continuous beams. In this study, the original MDM has been formulated in a closed form through a matrix formulation. Numerical results are presented to demonstrate the accuracy and efficiency of the proposed formulation.

\section{The Moment Distribution Method}

The MDM relies on a series of calculations that are repeated until every cycle comes closer to the final situation. In this way, it is possible to avoid solving simultaneous algebraic equations. This method is a unique method of structural analysis, in which the solution is obtained iteratively without even formulating the equations for the unknowns. The following beam will be used to illustrate the method.

In the first stage of Figure 1, rotation is possible at both $B$ and $C$. The second stage is that rotation at $B$ and $C$ is prevented, and the load is applied obtaining the initial moments. Afterwards, in the third stage, allow $B$ to rotate until moment equilibrium is reached and then rotation at $B$ will induce a moment at $C$. Finally, in the fourth stage of Figure 1, allow $C$ to rotate until moment equilibrium is reached. The rotation of $C$ will induce a moment at $B$. This process is repeated until moment equilibrium is reached at all nodes.

Assume that the sum of the initial moments at the node $B$ is equal to $M_{0}$. Rotation will take place until moment equilibrium is attained, that is, $\sum M_{B}=0$ (Figure 2).

Therefore,

$$
M_{B A}^{D}+M_{B C}^{D}+M_{0}=0,
$$

where $M_{B A}^{D}$ and $M_{B C}^{D}$ are the moments as a result of the rotation at $B$ and are called the distribution moments. Remember that all the other rotations and sway are fixed. Using the equations from the slope-deflection method $[4,5]$, it is easy to demonstrate that

$$
\begin{aligned}
& M_{B A}^{D}=\frac{2 E I_{A B}}{L_{A B}}\left(2 \theta_{B}\right)=\frac{4 E I_{A B}}{L_{A B}} \theta_{B}, \\
& M_{B C}^{D}=\frac{2 E I_{B C}}{L_{B C}}\left(2 \theta_{B}\right)=\frac{4 E I_{B C}}{L_{B C}} \theta_{B},
\end{aligned}
$$

where $E I$ is the cross-section rigidity, $L$ is the member length, and $\theta_{B}$ is the rotation at $B$.

Applying (2) into (1) and solving for $\theta_{B}$, the rotation at $B$ can be written as

$$
\theta_{B}=-\frac{M_{0}}{4 E I_{A B} / L_{A B}+4 E I_{B C} / L_{B C}} .
$$

Finally, the distribution moments are obtained, as follows:

$$
\begin{aligned}
M_{B A}^{D} & =-\frac{\left(4 E I_{A B} / L_{A B}\right) M_{0}}{4 E I_{A B} / L_{A B}+4 E I_{B C} / L_{B C}} \\
& =-\frac{k_{B A} M_{0}}{k_{B A}+k_{B C}}=-\frac{k_{B A} M_{0}}{\sum k_{B}}, \\
M_{B C}^{D} & =-\frac{\left(4 E I_{B C} / L_{B C}\right) M_{0}}{4 E I_{A B} / L_{A B}+4 E I_{B C} / L_{B C}} \\
& =-\frac{k_{B C} M_{0}}{k_{B A}+k_{B C}}=-\frac{k_{B C} M_{0}}{\sum k_{B}},
\end{aligned}
$$

where $k_{B A}$ is the stiffness of the member $B A$ at node $B$. It is also the moment that would be induced if a unit rotation was applied at $B$ in the member $B A$ and the rotation at $A$ was zero. If $B$ rotates, a bending moment will be induced at $A$ and $C$. Assuming a rotation $\theta_{B}$, the moment at $A$ can be written as follows:

$$
M_{A B}^{D}=-\frac{\left(2 E I_{A B} / L_{A B}\right) M_{0}}{4 E I_{A B} / L_{A B}+4 E I_{B C} / L_{B C}} .
$$

For this case, the distributed bending moment is half the value of the distributed bending moment at $B$. This is called the carry-over factor, $C_{B A}=0,5$. It should be mentioned that stiffness and carry-over factors may vary with several parameters, depending on the shape of the structural member, the restraint conditions, and the structural effects considered. 
In summary, the MDM still remains an important tool for analysis of structures in practice and can be programmed for complicated problems without the use of modern structural analysis software. At the first stage of the analysis, it is assumed that the rigid joints of the structural members are initially fixed against rotations as in the displacement method. The reactive moments produced by external loads are computed. These moments are unbalanced at the joints of the original nonrestrained structure. In order to equilibrate the joints the moments are distributed proportionally to the corresponding member stiffness. These distributed moments are associated with the so-called carry-over moments at the opposite ends of structural members. They are considered to be new incremental unbalanced moments, and the procedure repeats until the unbalanced moments become negligible. The final moments at the ends of all members are the sum of all distributed moment increments. This procedure assumes the joint rotations only.

According to Volokh [6], the MDM is nothing but Jacobi iterative scheme in disguise. It is shown that the method is the incremental form of Jacobi iterative scheme applied to the classical displacement formulation of the problem.

\section{Sign Convention}

The MDM convention that the final moments at a joint or a support are considered to be acting from the joint or support to the member is used. The convention adopted here is easier for moments and just as easy for forces, so its use is recommended. The positive direction for final moments is taken as clockwise.

The use of a rotational sense to determine the sign of final moments automatically arranges that the algebraic sum of such moments equates to zero, at any joint in equilibrium. The clockwise direction also implies the use of the fourth quadrant for the definition of positive linear directions. Thus, forces, distances, and linear displacements are taken as positive when measured from left to right or vertically downwards. This is in accordance with the positive directions used in beam theory [7].

This sign convention is convenient when considering joint equilibrium in the slope-deflection and moment distribution methods. For a horizontal beam subjected to downward loading, the MDM convention gives negative and positive fixed-end moment at the left-hand and right-hand supports, respectively.

\section{Matrix Formulation}

This section reports some of the basic relations of the proposed formulation for the MDM based on a matrix notation. Let the distribution and carry-over factors and the initial moments be written as follows:

$$
\begin{aligned}
\{M\}= & \left\{M_{0}\right\}+[\beta]\left\{M_{0}\right\}+[\alpha][\beta]\left\{M_{0}\right\}+[\beta][\alpha][\beta]\left\{M_{0}\right\} \\
& +[\alpha][\beta][\alpha][\beta]\left\{M_{0}\right\}+[\beta][\alpha][\beta][\alpha][\beta]\left\{M_{0}\right\}+\cdots,
\end{aligned}
$$

where $\{M\}$ is a vector which contains the final moments at the ends of the members and dimension $(m \times 1),\left\{M_{0}\right\}$ is also a column vector which contains the fixed-ends moments of the members and dimension $(m \times 1),[\beta]$ is a square matrix which contains the distribution factors and dimension $(m \times$ $m),[\alpha]$ is a square matrix which contains the carry-over factors and dimension $(m \times m)$, and, finally, $m$ is the number of the structural members multiplied by two.

Note that the first term of the series represented by (6) is the vector which contains the fixed-ends moments of the members. The second term is a product between the distribution factors and the fixed-ends moments, obtaining a new set of moments when all nodes of the structure are released simultaneously. The third term of the series is the product between the distribution factors and the set of moments obtained previously, and the series continues for $n$ cycles. Equation (6) can be rewritten as

$$
\begin{aligned}
\{M\}= & ([I]+[\beta]+[\alpha][\beta]+[\beta][\alpha][\beta]+[\alpha][\beta][\alpha][\beta] \\
& +[\beta][\alpha][\beta][\alpha][\beta]+\cdots)\left\{M_{0}\right\},
\end{aligned}
$$

where $[I]$ is a simplest nontrivial diagonal matrix known as identity matrix. After $n$ cycles of the method and using some properties of the identity matrix, the following equation is obtained:

$$
\begin{gathered}
\{M\}=\{[I]+[\beta]\}\left\{[I]+[\alpha][\beta]+([\alpha][\beta])^{2}+([\alpha][\beta])^{3}\right. \\
\left.+\cdots+([\alpha][\beta])^{n-1}\right\}\left\{M_{0}\right\}
\end{gathered}
$$

or

$$
\{M\}=\{[I]+[\beta]\}\left\{S_{n}\right\}\left\{M_{0}\right\},
$$

where $\left\{S_{n}\right\}=\left\{[I]+[\alpha][\beta]+([\alpha][\beta])^{2}+([\alpha][\beta])^{3}+\cdots+\right.$ $\left.([\alpha][\beta])^{n-1}\right\}$. Since a geometric progression series is a sum of terms in which two successive terms always have the same ratio, $\left\{S_{n}\right\}$ is a geometric progression with a common ratio of $r=\{[\alpha][\beta]\}$.

The sum of the first $n$ terms of a geometric progression is given by

$$
\left\{S_{n}\right\}=\frac{a_{1}\left(1-r^{n}\right)}{(1-r)},
$$

where $a_{1}$ is the first term and $r$ is the ratio of the series.

Substituting $[I]$ as the first term and $[\alpha][\beta]$ as the ratio of the series into (10) gives

$$
\left\{S_{n}\right\}=\frac{[I]\left\{[I]-([\alpha][\beta])^{n}\right\}}{\{[I]-[\alpha][\beta]\}} .
$$

Note that the terms in matrix $[\beta]$ are less than the unity. Therefore, when $n$ in the term $([\alpha][\beta])^{n}$ tends to the infinite, $\left\{S_{n}\right\}$ can be written as

$$
\left\{S_{n}\right\}=\frac{[I]}{\{[I]-[\alpha][\beta]\}} \quad \text { or } \quad\left\{S_{n}\right\}=\{[I]-[\alpha][\beta]\}^{-1} .
$$




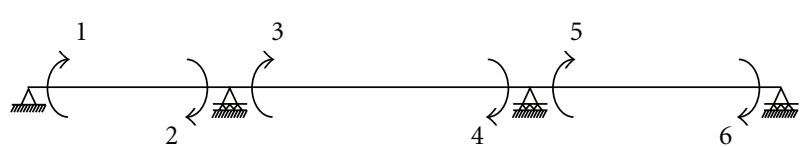

FIgURE 3: Reference coordinates of the structure.

Substituting (12) into (9) gives

$$
\{M\}=\{[I]+[\beta]\}\{[I]-[\alpha][\beta]\}^{-1}\left\{M_{0}\right\},
$$

which represents the closed form of the MDM through a matrix formulation.

\section{Reference Coordinates}

The analysis of a continuous beam by the closed form of the MDM can be achieved, as previously demonstrated, by solving (13) which is expressed in a matrix form. The development of the matrix elements of the latter equation must be described with respect to the reference coordinates. It is necessary to identify the ends of the members as shown in Figure 3.

In the case of matrix $[\beta]$ which represents the distribution factors, it is observed that in the MDM the distribution factor at the end of member corresponding to reference coordinate 2 will multiply the bending moments associated to reference coordinates 2 and 3 . Thus, the distribution factor has to be repeated two times in line 2 of the matrix and located in columns 2 and 3. The distribution factor corresponding to reference coordinate 5 will multiply the bending moments associated to reference coordinates 4 and 5. This distribution factor will be repeated two times in line 5 of the matrix, and located in columns 4 and 5 . In other words, the reference coordinate of each distribution factor defines the line of the matrix and its value is repeated as many times according to the number of the end members in a node. The location in the columns is defined by the reference coordinates of the end members, while the other values are zero.

In this way, the matrix $[\beta]$ for the continuous beam in Figure 3 is given by

$$
[\beta]=\left[\begin{array}{cccccc}
-\beta_{1} & 0 & 0 & 0 & 0 & 0 \\
0 & -\beta_{2} & -\beta_{2} & 0 & 0 & 0 \\
0 & -\beta_{3} & -\beta_{3} & 0 & 0 & 0 \\
0 & 0 & 0 & -\beta_{4} & -\beta_{4} & 0 \\
0 & 0 & 0 & -\beta_{5} & -\beta_{5} & 0 \\
0 & 0 & 0 & 0 & 0 & -\beta_{6}
\end{array}\right]_{6 \times 6}
$$

In order to evaluate the matrix $[\alpha]$ in Figure 3, which represents the carry-over factors, consider, for instance, the case at the end of the member corresponding to reference coordinate 2 . The carry-over factor will multiply the bending moments associated to reference coordinate 2 , but the result will be added to the bending moments associated to reference coordinate 1 . Therefore, this carry-over factor will be located in column 2 and line 1 in matrix $[\alpha]$. Similarly, the carry-over factor corresponding to reference coordinate 5 will multiply the bending moments associated to reference coordinates 5 , but the result will be added to the bending moments associated to reference coordinate 6 . This carry-over factor will be located in column 5 and line 6 in matrix $[\alpha]$. To summarize the procedure, in the case of the carry-over factors, the member's reference coordinate identifies the column in which the correspondent value will be located and the opposite reference coordinate identifies the line of the matrix, while the other values are zero.

Applying this procedure in the matrix of the carry-over factors leads to

$$
[\alpha]=\left[\begin{array}{cccccc}
0 & \alpha_{2} & 0 & 0 & 0 & 0 \\
\alpha_{1} & 0 & 0 & 0 & 0 & 0 \\
0 & 0 & 0 & \alpha_{4} & 0 & 0 \\
0 & 0 & \alpha_{3} & 0 & 0 & 0 \\
0 & 0 & 0 & 0 & 0 & \alpha_{6} \\
0 & 0 & 0 & 0 & \alpha_{5} & 0
\end{array}\right]_{6 \times 6} .
$$

After all of the required matrices have been obtained, some of them with respect to the chosen reference coordinates, the closed form of the MDM can be done.

\section{Numerical Examples}

To compare the performance of the proposed formulation with the traditional one previously reported in the literature, four numerical examples are presented.

The first example is a continuous beam shown in Figure 4 given by Süssekind [8]. This beam is selected as a benchmark problem in the present study. In this particular, example certain features should be noted. First, the support at $A$ is a fixed one and the support at $E$ is an outside pinned support, so that the final moment at $E$ must be zero. The loading and the span lengths are given in Figure 4. As seen before, it is necessary to identify the ends of the members, with respect to reference coordinates, as shown in Figure 5.

The fixed-end moments of the members and the distribution and carry-over factors are calculated from the elementary procedures of structural analysis. These values are given in Table 1 as follows.

Using the values from Table 1 and putting into a matrix form, the matrices $[\beta]$ and $[\alpha]$ for the continuous beam in Figure 4 are given, respectively, by

$$
[\beta]=\left[\begin{array}{cccccccc}
0 & 0 & 0 & 0 & 0 & 0 & 0 & 0 \\
0 & -0.67 & -0.67 & 0 & 0 & 0 & 0 & 0 \\
0 & -0.33 & -0.33 & 0 & 0 & 0 & 0 & 0 \\
0 & 0 & 0 & -0.5 & -0.5 & 0 & 0 & 0 \\
0 & 0 & 0 & -0.5 & -0.5 & 0 & 0 & 0 \\
0 & 0 & 0 & 0 & 0 & -0.57 & -0.57 & 0 \\
0 & 0 & 0 & 0 & 0 & -0.43 & -0.43 & 0 \\
0 & 0 & 0 & 0 & 0 & 0 & 0 & -1
\end{array}\right]_{8 \times 8}
$$


TABLE 1: Initial moments at the ends of the members and the distribution and carry-over factors for the first example.

\begin{tabular}{lcccccccc}
\hline Node & $A$ & \multicolumn{2}{c}{$B$} & & $C$ & & $D$ & \\
Members & $A B$ & $B A$ & $B C$ & $C B$ & $C D$ & $D C$ & $D E$ \\
\hline Fixed-end moments & -4 & 4 & -4 & 4 & -4 & 4 & -7.5 \\
Distribution factors & 0 & 0.67 & 0.33 & 0.5 & 0.5 & 0.57 & 0.43 \\
Carry-over factors & 0.5 & 0.5 & 0.5 & 0.5 & 0.5 & 0.5 & 0.5 \\
\hline
\end{tabular}

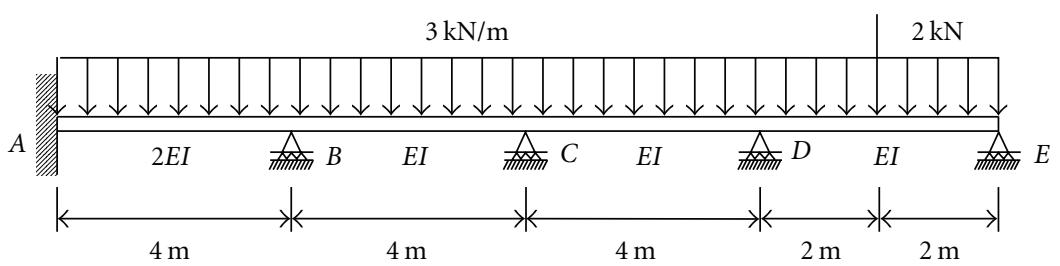

FIgURE 4: Continuous beam for the first example.

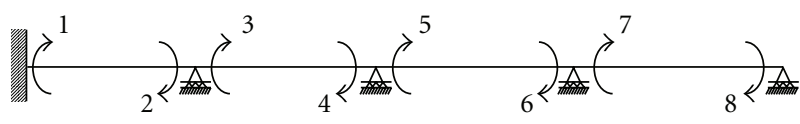

FIGURE 5: Reference coordinates for the first example.

$$
[\alpha]=\left[\begin{array}{cccccccc}
0 & 0.5 & 0 & 0 & 0 & 0 & 0 & 0 \\
0.5 & 0 & 0 & 0 & 0 & 0 & 0 & 0 \\
0 & 0 & 0 & 0.5 & 0 & 0 & 0 & 0 \\
0 & 0 & 0.5 & 0 & 0 & 0 & 0 & 0 \\
0 & 0 & 0 & 0 & 0 & 0.5 & 0 & 0 \\
0 & 0 & 0 & 0 & 0.5 & 0 & 0 & 0 \\
0 & 0 & 0 & 0 & 0 & 0 & 0 & 0.5 \\
0 & 0 & 0 & 0 & 0 & 0 & 0 & 0
\end{array}\right]_{8 \times 8}
$$

Using (13), which represents the closed form of the MDM through a matrix formulation, leads to the following final moments at the ends of the members:

$$
\begin{array}{cc}
M_{1}=-3.91 \mathrm{kN} \cdot \mathrm{m}, & M_{2}=4.19 \mathrm{kN} \cdot \mathrm{m}, \\
M_{3}=-4.19 \mathrm{kN} \cdot \mathrm{m}, & M_{4}=3.48 \mathrm{kN} \cdot \mathrm{m}, \\
M_{5}=-3.48 \mathrm{kN} \cdot \mathrm{m} ; & M_{6}=5.87 \mathrm{kN} \cdot \mathrm{m}, \\
M_{7}=-5.87 \mathrm{kN} \cdot \mathrm{m}, & M_{8}=0 \mathrm{kN} \cdot \mathrm{m} .
\end{array}
$$

The numerical results obtained with the matrix formulation were compared with the results obtained in the literature to illustrate the applicability and accuracy of the proposed formulation. All the final moments at the ends of the members were exactly the same as given by Süssekind [8].

In the second example, the behavior of the matrix formulation was tested in a continuous nonprismatic beam shown in Figure 6 given by R. C. Lopes and A. P. Lopes [9]. This beam has four supports at $A, B, C$, and $D$. The loading and the span lengths are also given in Figure 6, and the reference coordinates are given in Figure 7.

The initial moments at the ends of the members and the distribution and carry-over factors are given in Table 2.

Using the values from Table 2 and putting into a matrix form, the matrices $[\beta]$ and $[\alpha]$ for the continuous beam in Figure 6 are given, respectively, by

$$
\begin{gathered}
{[\beta]=\left[\begin{array}{cccccc}
-1 & 0 & 0 & 0 & 0 & 0 \\
0 & -0.875 & -0.875 & 0 & 0 & 0 \\
0 & -0.125 & -0.125 & 0 & 0 & 0 \\
0 & 0 & 0 & -0.125 & -0.125 & 0 \\
0 & 0 & 0 & -0.875 & -0.875 & 0 \\
0 & 0 & 0 & 0 & 0 & -1
\end{array}\right]_{6 \times 6},} \\
{[\alpha]=\left[\begin{array}{cccccc}
0 & 0.680 & 0 & 0 & 0 & 0 \\
0.372 & 0 & 0 & 0 & 0 & 0 \\
0 & 0 & 0 & 0.637 & 0 & 0 \\
0 & 0 & 0.637 & 0 & 0 & 0 \\
0 & 0 & 0 & 0 & 0 & 0.372 \\
0 & 0 & 0 & 0 & 0.680 & 0
\end{array}\right]_{6 \times 6},}
\end{gathered}
$$

Using (13), which represents the closed form of the MDM through a matrix formulation, leads to the following final moments at the ends of the members:

$$
\begin{gathered}
M_{1}=0 \mathrm{kN} \cdot \mathrm{m}, \quad M_{2}=226.65 \mathrm{kN} \cdot \mathrm{m}, \\
M_{3}=-226.65 \mathrm{kN} \cdot \mathrm{m}, \quad M_{4}=333.43 \mathrm{kN} \cdot \mathrm{m}, \\
M_{5}=-333.43 \mathrm{kN} \cdot \mathrm{m}, \quad M_{6}=0 \mathrm{kN} \cdot \mathrm{m} .
\end{gathered}
$$

The numerical results obtained with the matrix formulation were compared with a commercial structuralprogram 
TABLE 2: Initial moments at the ends of the members and the distribution and carry-over factors for the second example.

\begin{tabular}{lcccccc}
\hline Node & $A$ & \multicolumn{2}{c}{$B$} & & $C$ & \\
Members & $A B$ & $B A$ & $B C$ & $C B$ & $C D$ & -78.17 \\
\hline Fixed-end moments & -215.11 & 78.17 & -218.40 & 358.60 & 0.125 & 0.875 \\
Distribution factors & 1 & 0.875 & 0.125 & 0.637 & 0.680 \\
Carry-over factors & 0.372 & 0.680 & & 0.637 & 0.372 \\
\hline
\end{tabular}

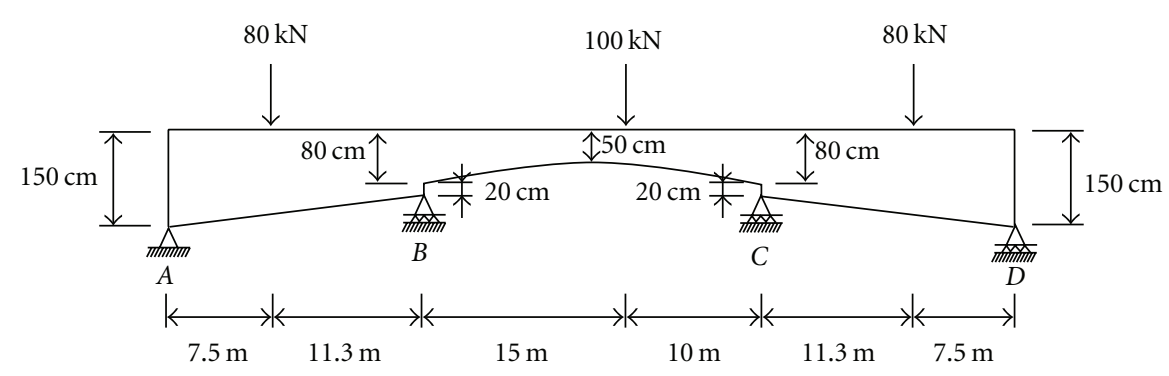

Figure 6: Continuous beam for the second example.

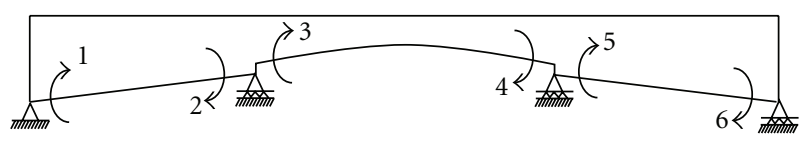

FIGURE 7: Reference coordinates for the second example.

known as SAP2000 v. 14.1.0. The matrix formulation yields excellent results.

In the third example, the proposed formulation was tested in a symmetric continuous beam shown in Figure 8 given by Leet et al. [10]. This beam has five supports at $A, B, C, D$, and $E$. Supports $A$ and $B$ are fixed, and the flexural stiffness is assumed to be constant. The loading and the span lengths are also given in Figure 8, and the reference coordinates are given in Figure 9.

The fixed-end moments of the members and the distribution and carry-over factors are calculated from the elementary procedures of structural analysis. These values are given in Table 3 as follows.

Using the values from Table 3 and putting into a matrix form, the matrices $[\beta]$ and $[\alpha]$ for the continuous beam in Figure 8 are given, respectively, by

$$
[\beta]=\left[\begin{array}{cccccccc}
0 & 0 & 0 & 0 & 0 & 0 & 0 & 0 \\
0 & -0.43 & -0.43 & 0 & 0 & 0 & 0 & 0 \\
0 & -0.57 & -0.57 & 0 & 0 & 0 & 0 & 0 \\
0 & 0 & 0 & -0.5 & -0.5 & 0 & 0 & 0 \\
0 & 0 & 0 & -0.5 & -0.5 & 0 & 0 & 0 \\
0 & 0 & 0 & 0 & 0 & -0.57 & -0.57 & 0 \\
0 & 0 & 0 & 0 & 0 & -0.43 & -0.43 & 0 \\
0 & 0 & 0 & 0 & 0 & 0 & 0 & 0
\end{array}\right]_{8 \times 8},
$$

$$
[\alpha]=\left[\begin{array}{cccccccc}
0 & 0.5 & 0 & 0 & 0 & 0 & 0 & 0 \\
0.5 & 0 & 0 & 0 & 0 & 0 & 0 & 0 \\
0 & 0 & 0 & 0.5 & 0 & 0 & 0 & 0 \\
0 & 0 & 0.5 & 0 & 0 & 0 & 0 & 0 \\
0 & 0 & 0 & 0 & 0 & 0.5 & 0 & 0 \\
0 & 0 & 0 & 0 & 0.5 & 0 & 0 & 0 \\
0 & 0 & 0 & 0 & 0 & 0 & 0 & 0.5 \\
0 & 0 & 0 & 0 & 0 & 0 & 0.5 & 0
\end{array}\right]_{8 \times 8}
$$

Using (13), which represents the closed form of the MDM through a matrix formulation, leads to the following final moments at the ends of the members:

$$
\begin{array}{ll}
M_{1}=-234.28 \mathrm{kN} \cdot \mathrm{m}, & M_{2}=150.11 \mathrm{kN} \cdot \mathrm{m}, \\
M_{3}=-150.11 \mathrm{kN} \cdot \mathrm{m}, & M_{4}=38.38 \mathrm{kN} \cdot \mathrm{m}, \\
M_{5}=-38.38 \mathrm{kN} \cdot \mathrm{m}, & M_{6}=150.11 \mathrm{kN} \cdot \mathrm{m}, \\
M_{7}=-150.11 \mathrm{kN} \cdot \mathrm{m}, & M_{8}=234.28 \mathrm{kN} \cdot \mathrm{m} .
\end{array}
$$

The numerical results obtained with the matrix formulation were compared with a commercial structural program known as SAP2000 v. 14.1.0. The bending moment diagram is plotted in Figure 10. The matrix formulation yields excellent results, and the minor differences less than $1 \%$ are due to shear deformations included in the analysis via SAP2000.

In the fourth example, the proposed formulation was tested in an asymmetric continuous beam shown in Figure 8 
TABLE 3: Initial moments at the ends of the members and the distribution and carry-over factors for the third example.

\begin{tabular}{lcccccccc}
\hline Node & $A$ & \multicolumn{2}{c}{$B$} & & $C$ & & $D$ & $E$ \\
Members & $A B$ & $B A$ & $B C$ & $C B$ & $C D$ & $D C$ & $D E$ & $E D$ \\
\hline Fixed-end moments & -206.225 & 206.225 & -75.625 & 75.625 & -75.625 & 75.625 & -206.225 \\
Distribution factors & 0 & 0.43 & 0.57 & 0.5 & 0.5 & 0.57 & 0.43 \\
Carry-over factors & 0.5 & 0.5 & 0.5 & 0.5 & 0.5 & 0.5 & 0.5 \\
\hline
\end{tabular}

TABLE 4: Initial moments at the ends of the members and the distribution and carry-over factors for the fourth example.

\begin{tabular}{lccccc}
\hline Node & $A$ & & $B$ & & $C$ \\
Members & $A B$ & $B A$ & $B C$ & $C B$ & 133.225 \\
\hline Fixed-end moments & -63.875 & 63.875 & -133.225 & 0.33 & 0 \\
Distribution factors & 0 & 0.5 & 0.5 & 0.5 & 0.67 \\
Carry-over factors & 0.5 & 0.5 & 0.5 & 0.5 \\
\hline
\end{tabular}

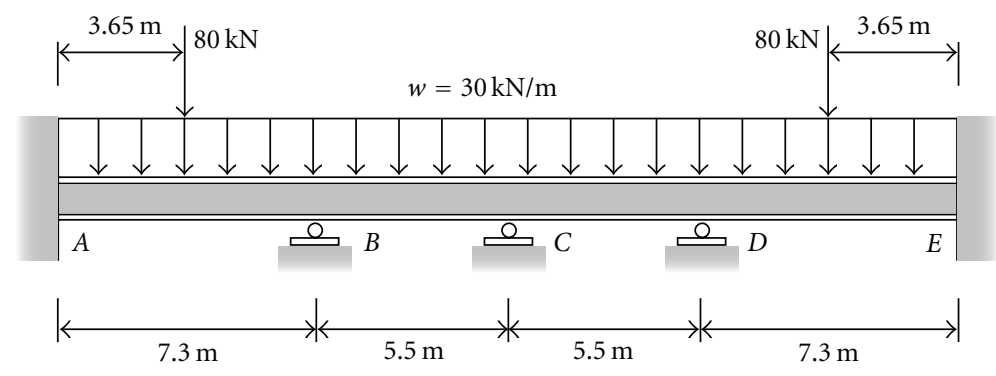

FIgURE 8: Continuous beam for the third example.

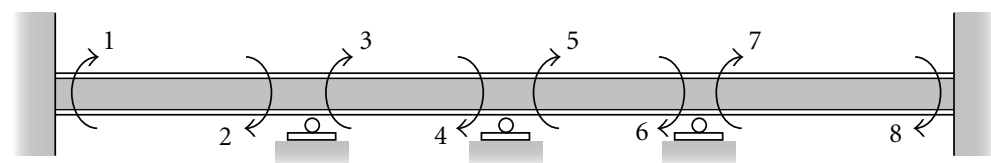

FIgURE 9: Reference coordinates for the third example.

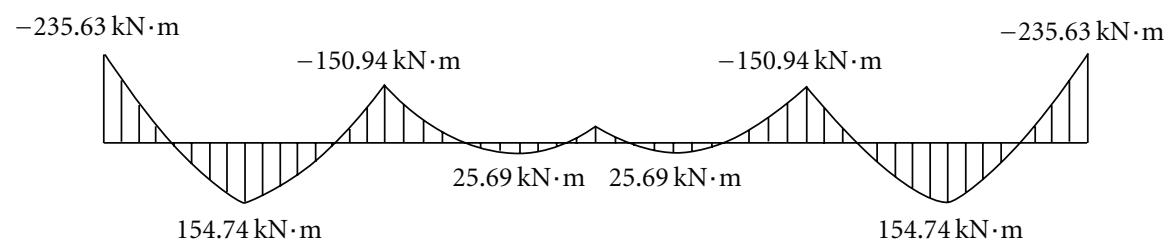

FIGURE 10: Bending moment diagram for the third example.

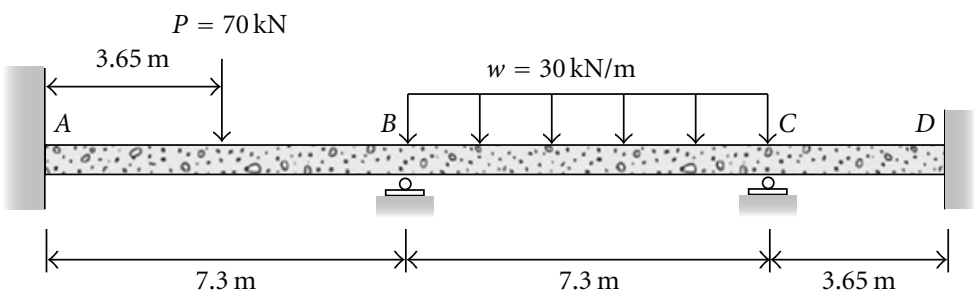

FIgURE 11: Continuous beam for the fourth example. 


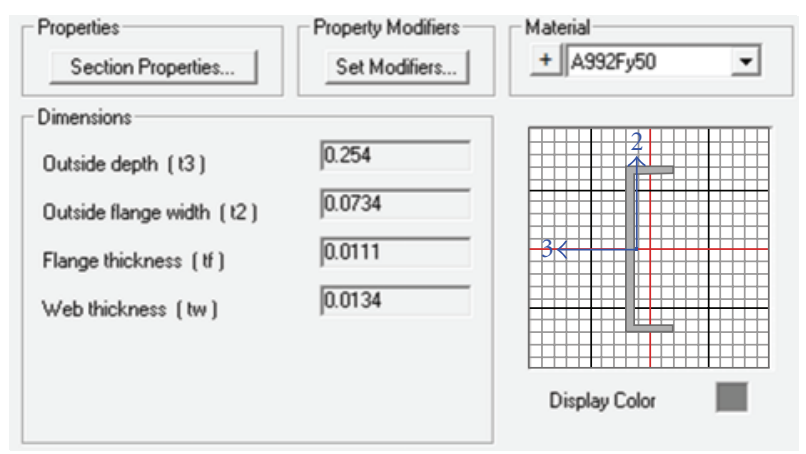

FIGURE 12: Section properties for the fourth example.

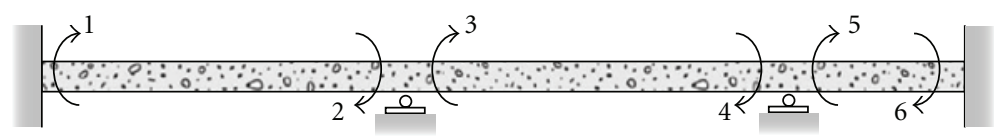

FIGURE 13: Reference coordinates for the fourth example.

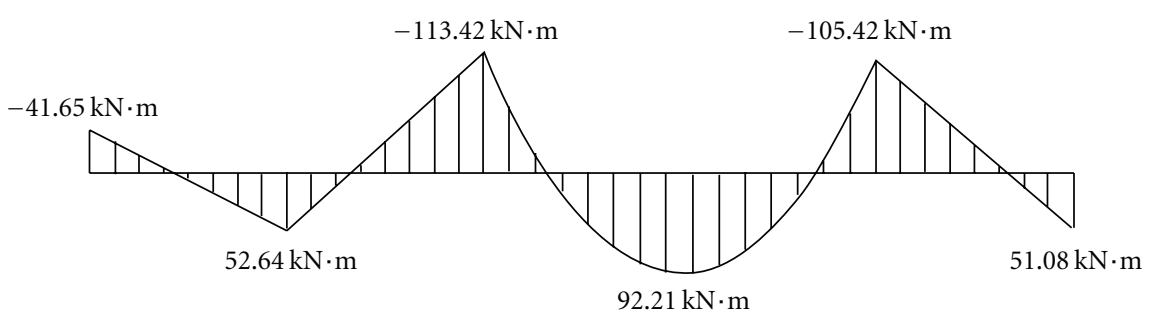

FIgURE 14: Bending moment diagram for the fourth example.

given by Leet et al. [10]. This beam has four supports at $A, B$, $C$, and $D$. Supports $A$ and $B$ are fixed and the cross-section of the beam is a C-Shape $(\mathrm{C} 10 \times 25)$ given by the AISC steel construction manual [11]. The loading and the span lengths are also given in Figure 11, the section properties are given in Figure 12, and the reference coordinates are given in Figure 13.

The initial moments at the ends of the members and the distribution and carry-over factors are given in Table 4 .

Using the values from Table 4 and putting into a matrix form, the matrices $[\beta]$ and $[\alpha]$ for the continuous beam in Figure 6 are given, respectively, by

$$
[\beta]=\left[\begin{array}{cccccc}
0 & 0 & 0 & 0 & 0 & 0 \\
0 & -0.5 & -0.5 & 0 & 0 & 0 \\
0 & -0.5 & -0.5 & 0 & 0 & 0 \\
0 & 0 & 0 & -0.33 & -0.33 & 0 \\
0 & 0 & 0 & -0.67 & -0.67 & 0 \\
0 & 0 & 0 & 0 & 0 & 0
\end{array}\right]_{6 \times 6}
$$

$$
[\alpha]=\left[\begin{array}{cccccc}
0 & 0.5 & 0 & 0 & 0 & 0 \\
0.5 & 0 & 0 & 0 & 0 & 0 \\
0 & 0 & 0 & 0.5 & 0 & 0 \\
0 & 0 & 0.5 & 0 & 0 & 0 \\
0 & 0 & 0 & 0 & 0 & 0.5 \\
0 & 0 & 0 & 0 & 0.5 & 0
\end{array}\right]_{6 \times 6}
$$

Using (13), which represents the closed form of the MDM through a matrix formulation, leads to the following final moments at the ends of the members:

$$
\begin{array}{ll}
M_{1}=-40.06 \mathrm{kN} \cdot \mathrm{m}, & M_{2}=111.51 \mathrm{kN} \cdot \mathrm{m}, \\
M_{3}=-111.51 \mathrm{kN} \cdot \mathrm{m}, & M_{4}=105.22 \mathrm{kN} \cdot \mathrm{m}, \\
M_{5}=-105.22 \mathrm{kN} \cdot \mathrm{m}, & M_{6}=-52.61 \mathrm{kN} \cdot \mathrm{m} .
\end{array}
$$

The numerical results obtained with the matrix formulation were compared with a commercial structural program known as SAP2000 v. 14.1.0. The bending moment diagram is plotted in Figure 13. The matrix formulation 
yields excellent results, and the minor differences less than $2 \%$ are due to shear deformations included in the analysis via SAP2000.

\section{Conclusions}

A new formulation for the MDM based on a matrix formulation has been developed. The main advantage of the proposed scheme is the use of only one equation to solve the problem without iteration. The results obtained here show that accurate values for the final moments at the ends of the members have been achieved compared to previous examples given in the literature or using a commercial structural program. It should be mentioned that the matrix formulation can be applied to framed structures too. Nowadays, it seems that the simpler possibilities of the MDM have all been discovered and that its economical use for the analysis and design of complex structures must now be assessed in relation to available electronic computing programs and facilities. From this point of view, the matrix formulation of the MDM emerges as an option to solve structural problems. It is apparent, therefore, that with research developments here and abroad the MDM still has many exciting possibilities to add to its considerable achievements. The next step of this research is to extend the proposed formulation to three-dimension problems and also the analysis of structures considering shear deformations.

\section{References}

[1] H. Cross, "Analysis of continuous frames by distributing fixedend moments," Proceedings of the American Society of Civil Engineers, vol. 96, pp. 919-928, 1930.

[2] H. Cross and N. D. Morgan, Continuous Frames of Reinforced Concrete, John Wiley \& Sons, New York, NY, USA, 1932.

[3] E. Lightfoot, Moment Distribution: A Rapid Method of Analysis for Rigid-Jointed Structures, Barnes \& Noble, New York, NY, USA, 1961.

[4] H. I. Laursen, Structural Analysis, McGraw-Hill, New York, NY, USA, 2nd edition, 1978.

[5] A. Kassimali, Structural Analysis, International Thomson Publishing, New York, NY, USA, 1995.

[6] K. Y. Volokh, "On foundations of the Hardy Cross method," International Journal of Solids and Structures, vol. 39, no. 16, pp. 4197-4200, 2002.

[7] S. P. Timoshenko and J. N. Goodier, Theory of Elasticity, McGraw-Hill, New York, NY, USA, 1980.

[8] J. C. Süssekind, Curso de Análise Estrutural, vol. 3, Editora da Universidade de São Paulo, São Paulo, Brasil, 1973.

[9] R. C. Lopes and A. P. Lopes, "Structural analysis: The moment distribution method" (Portuguese), In press.

[10] K. M. Leet, C. M. Uang, and A. M. Gilbert, Fundamentals of Structural Analysis, McGraw-Hill, New York, NY, USA, 4th edition, 2011.

[11] AISC, Steel Construction Manual, American Institute of Steel Construction, 13th edition, 2008. 

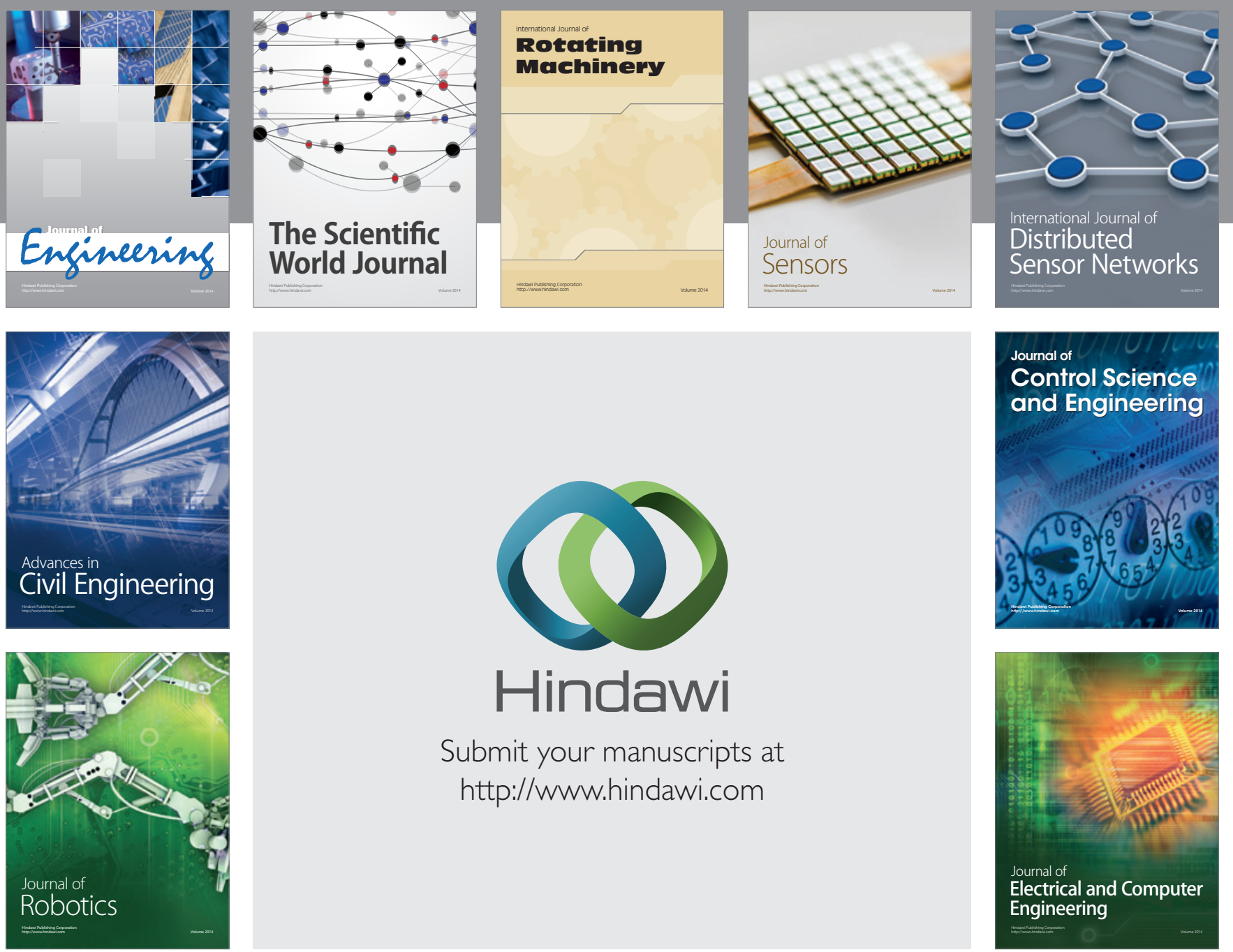

Submit your manuscripts at

http://www.hindawi.com
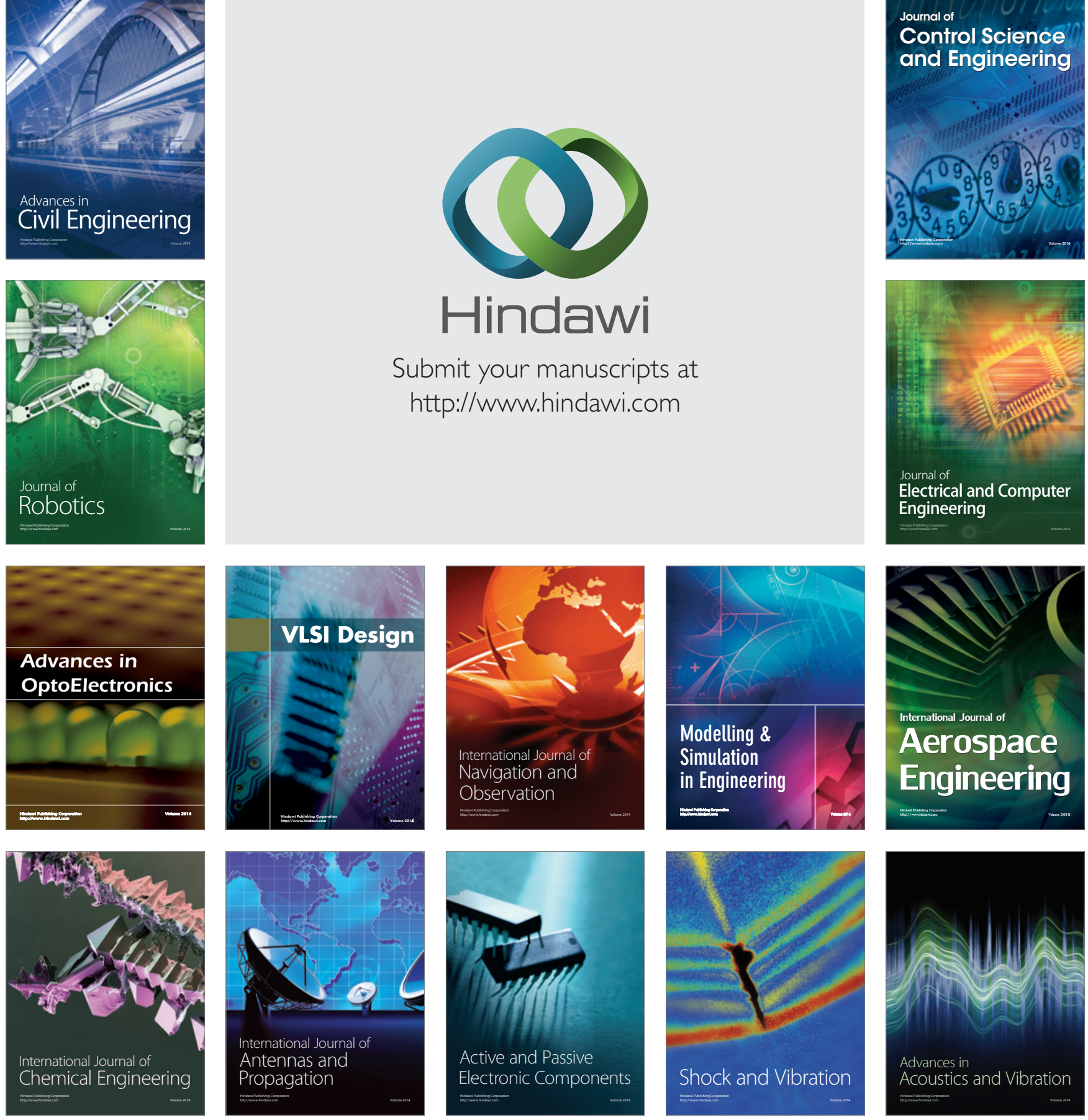